\title{
APPLICATION OF WELDING TECHNOLOGIES FOR SUPPRESSION OF LIQUATION IN LARGE INGOTS
}

\section{V.A. SHAPOVALOV}

E.O. Paton Electric Welding Institute, NASU

11 Kazimir Malevich Str., 03680, Kiev, Ukraine. E-mail: office@paton.kiev.ua

\begin{abstract}
The problem of liquation continuously attracts an attention of metallurgists and it is especially acute in producing of large ingots. Dendritic liquation can deteriorate the ingot so that it can occurr to be unsuitable for manufacture of large critical-purpose parts. It is shown that the main cause of liquation is a low rate of solidification. Its increase is impossible in melting of large ingots by using the traditional technologies. Therefore, one of the ways of suppression of the dendritic liquation is a stage-by-stage surfacing (formation) of ingots by using the welding technologies. The article gives the experimental data confirming the feasibility of the ingot structure control independently of its sizes. The fields of application of the suggested approach for manufacture of critical products are predicted. 10 Ref., 5 Figures.
\end{abstract}

Keywords: dendritic liquation, coefficient of distribution, rate of solidification, ESR, VAR, EBR, PAR, macro- and microstructure

Liquation is the non-homogeneity of chemical composition, occurred at its crystallization. There is dendritic and zonal liquation. The dendritic liquation for alloys results in decrease of corrosion resistance, reduction in ductility, formation of lineage structure in pressure treatment, decrease in solidus temperature, hazard of fusion of grain boundaries during heat treatment, instability of structure and properties of metal with time. Zonal liquation can be positive, negative, carbide, off-center (cord-type) and gravity. The cause of liquation is the different solubility of the alloying component or impurity in solid and liquid phases (Figure 1).

The ratio of concentration of impurities in a solid phase $\gamma C_{\mathrm{s}}$ to concentration of impurities in a liquid phase $C_{1}$ is the coefficient of distribution

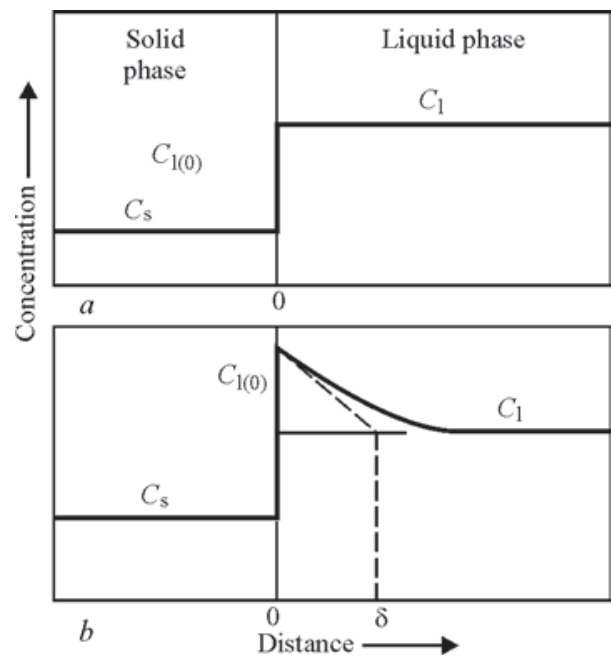

Figure 1. Scheme of distribution of impurities at the interface of solid and liquid phases in equilibrium $(a)$ and non-equilibrium $(b)$ conditions $(\delta$ - thickness of diffusion layer)

$$
K=\frac{C_{\mathrm{s}}}{C_{1}} .
$$

In metallurgical processes $K<1$ and depends on temperature and rate of solidification (for most alloying elements and impurities). At rapid (instantaneous) hardening $K=1$. At very slow solidification $K=K_{0}$, where $K_{0}$ is in equilibrium. With lower $K$ the liquation is stronger.

Usually, its value is given in handbooks for the equilibrium state.

Under the real conditions it is necessary to mention about the effective coefficient of distribution (Figure 2) which is determined by formula [1]

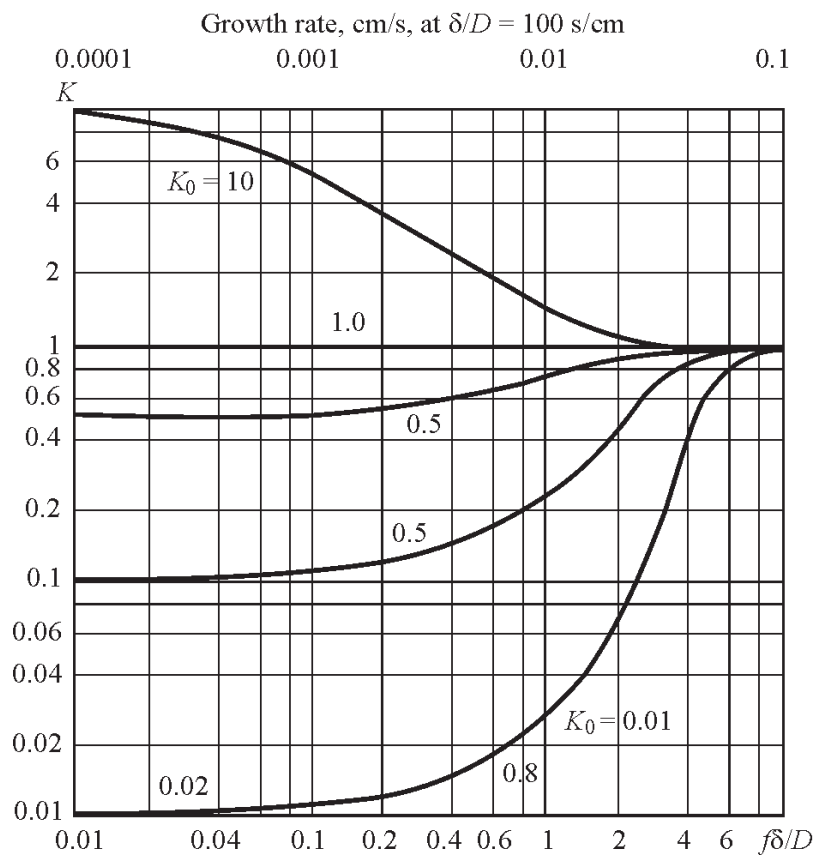

Figure 2. Effective coefficient of distribution versus value of given rate of movement of the solidified metal boundary [1] 


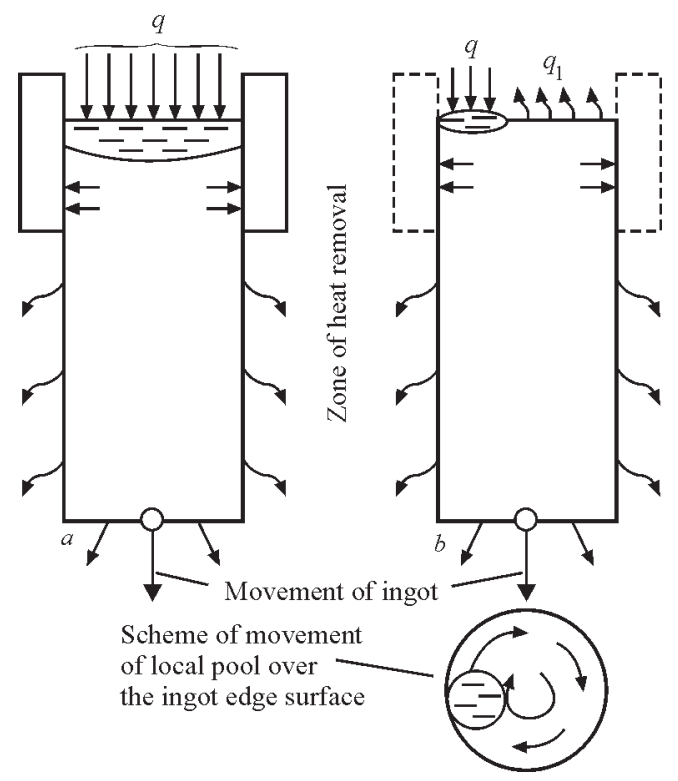

Figure 3. Scheme of supply and removal of heat from ingot: $a-$ diameters of pool and ingot are equal; $b$ - local metal pool

$$
K=\frac{K_{0}}{K_{0}+\left(1-K_{0}\right) e^{-f \delta / D}},
$$

where $f$ is solidification rate, $\mathrm{cm} / \mathrm{s} ; \delta$ is the thickness of diffusion layer adjacent to the front of crystallization and enriched with impurity, $\mathrm{cm}$; $D$ is the coefficient of impurity diffusion, $\mathrm{cm}^{2} / \mathrm{s}$.

The traditional technical procedures for prevention of the liquation are the following: control of temperature of poured metal; control of thermal field at the pool surface; adding of coolers; electromagnetic stirring, vibro-treatment; reverse rotation of metal; ultrasonic treatment; electrohydraulic pulse treatment; treatment of melt with immersed oscillating rod.

Unfortunately, the efficiency of traditional technical procedures is decreased with increase in the ingot weight. Therefore, the development of new methods
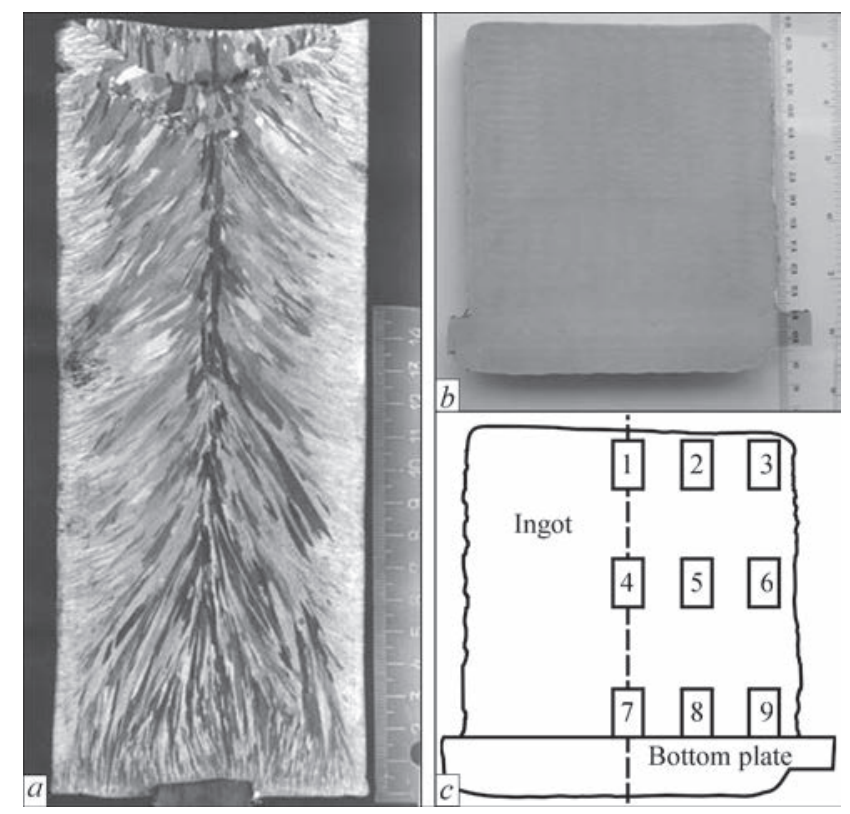

Figure 4. Microstructure of plasma-arc remelted ingot $(a)$ and ingot produced using layer-by-layer surfacing by local welding power source $(b)$, and map of specimens sampling $(c)$

of producing large ingots is urgent. The main cause of liquation is the low rate of solidification, and it is decreased non-linearly with increase in the ingot mass.

The way of the liquation prevention is the increase in the rate of solidification (crystallization) up to such a value which will provide the effective coefficient of distribution of impurities and alloying elements, which is $K \approx 1$.

The traditional methods of producing large ingots, including also the special metallurgy, cannot solve this problem. In case of exceeding the critical diameter of the ingot the crystallization, typical for VAR, ESR, EBR and PAR ingots of a small diameter, is violated and the processes lose their advantages [2-4]. The heat removal from the ingot middle downward and through a lateral surface is limited, and at the top

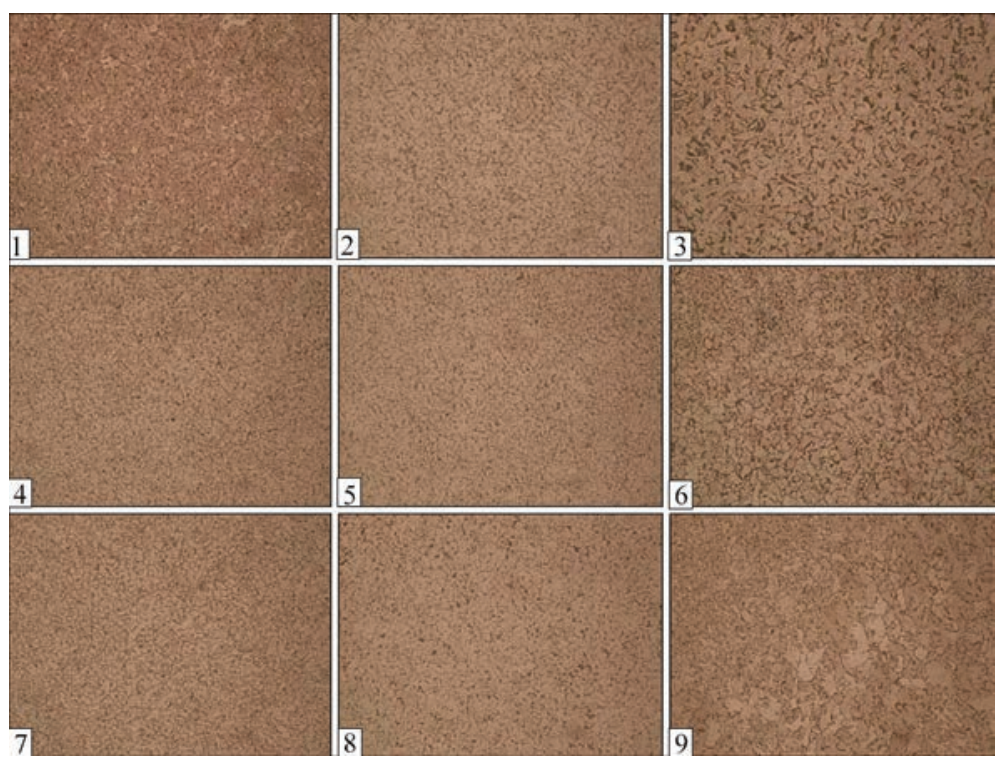

Figure 5. Microstructure $(\times 200)$ of specimens of ingot metal produced by increment during movement of small metal pool 
the overheated metal pool covers all the ingot cross section (Figure 3,a).

To solve the problem, i.e. the increase in solidification rate, it is necessary to organize the heat outflow. This possibility appears if the metal pool would have small sizes, and the rest top edge surface of the ingot would be solid with heat dissipation into the furnace atmosphere (Figure 3, $b$; flow $q_{1}$ ). Then, it would be possible to control the rate of crystallization and liquation processes by stirring the metal pool over the surface being surfaced at a high rate.

The problem of liquation can be solved by applying the welding technologies. Small weld pool by sizes and high speed of welding or surfacing, reaching several meters per minute, allows predicting the desired result with confidence. The welding processes are the most frequently applied in 3D-technologies for manufacture of products of the preset rather intricate shape. The processes of producing powerful turbine rotors are described in [5], the examples of prospects of manufacture of nuclear reactor bodies are given in [6]. The published works are devoted mainly to the shape formation, but there is negligible number of works, in which the attention is paid to the control of structure (liquation).

Our scientific interests belong to the area of structure control, first of all, and, secondly, to shape formation. Thus, by reducing the rate of movement of local metal pool to the area of small values $(\mathrm{mm} / \mathrm{min})$ and using a single-crystalline primer we produce the single crystals, the sizes of which are theoretically not limited [7, 8]. The crystals inherit structure and crystallographic orientation of the primer. With increase in the speed of welding source movement by 5-10 times as compared to rate of melting of ingots in known remelting processes, we can obtain random and rapid mass nucleation of crystals in a metal pool and, as a result, produce the fine-crystalline structure $[9,10]$. Convinced results about the wide possibilities of structure control as compared with traditional PAR are given in Figure 4 (when sizes of ingots are commensurable).

In PAR the ingot structure has two zones: zone of hardened crystals, and zone of large directed crystals, i.e. dendrites. Moreover, the transcrystallization is observed in the axial zone. In the ingot, produced as a result of its increment during movement of a small metal pool, a fine-grain structure is observed. Results of metallographic investigations show that the grain value is within the ranges of 8-9 (Figure 5). But, it is most important that the grain size does not almost depend on place of sampling the specimens from ingot: middle, top, or bottom. In realization of such a technological approach the effect of a scale factor on the ingot structure formation is eliminated, that, in its turn, opens up the great opportunities of increasing the service properties of the product, produced of such ingots, and leads to the metal saving as the scale factor is accepted to be equal to 1 , the value of which is usually decreased with increase in product dimensions. In addition, weight of the ingot can maximum approach the weight of the ready product, so, there is no need to cut out the bottom and head parts (this is one third of the large ingot weight).

The control of structure and liquation processes is rather important in manufacture of critical-purpose products, for example, discs of the aircraft engines. In practice, this can be replacement of a complicated and expensive powder technology.

\section{Conclusions}

1. Ineffective application of traditional technologies for control of liquation processes in crystallization of large ingots is shown.

2. Application of welding technologies for control of liquation processes is grounded.

3. Feasibility of producing homogeneous structure across the entire ingot section, including bottom and head parts, is confirmed.

4. Prospects of application of the developing method for melting of critical-purpose ingot and billets are shown.

1. (1961) Technology of semiconductor materials. Ed. by G. Bridgers et al. Moscow: Oborongiz.

2. Flemings, M. (1977) Solidification processing. Ed. by A.A. Zhukov. Moscow: Mir.

3. Nekhenzi, Yu.A. (1948) Steel casting. Moscow: GINTL ChTsM.

4. Mitchell, A., Belestain, A.S. (1985) Factors influencing temperature and solidification of ingots in ESR. Elektroshlak. Pereplav, Issue 6, 192-198.

5. Datta, R., Million, K., Zimmermann, H. (2003) Shape welding with MnMoNi alloys for heavy components. Welding and Cutting, 55(4), 216-224.

6. Diltey, U., Stein, I., Berger, C. et al. (2006) Future prospects of shape welding. Ibid., 5(3), 164-172.

7. Shapovalov, V.A., Latash, Yu.V., Zholud, V.V. et al. (2001) Large flat tungsten and molybdenum single crystals and methods of its production. In: Proc. of $7^{\text {th }}$ Int. Symp. on Pure Metals (Kharkov, 2001), 39-42.

8. Shapovalov, V.A., Latash, Yu.V., Borimskaya, S.T. et al. (2001) Oriented tungsten single crystals: Producing, examination and application. Metally, 1, 6-64.

9. Shapovalov, V.A., Grigorenko, G.M. (2015) Suppression of liquation processes in large ingots. Sovr. Elektrometallurgiya, 1, 26-30.

10. Shapovalov, V.A., Tsykulenko, K.A., Shapovalov, E.V. et al. (2015) Formation of large ingot by method of moving of local metal pool. Elektrometallurgiya, 2, 36-43. 